\title{
Experimental and modeling studies of a micro direct methanol fuel cell
}

\author{
D.S. Falcão ${ }^{a, *}$, V.B. Oliveira ${ }^{a}$, C.M. Rangel ${ }^{\text {b }}$, A.M.F.R. Pinto ${ }^{a, *}$ \\ a CEFT, Departamento de Engenharia Química, Faculdade de Engenharia da Universidade do Porto, Rua Dr. Roberto Frias, S/n, 4200-465 Porto, Portugal \\ ${ }^{\mathrm{b}}$ LNEG, Laboratório Nacional de Energia e Geologia, Fuel Cells and Hydrogen, Paço do Lumiar, 22, 1649-038 Lisboa, Portugal
}

\section{A R T I C L E I N F O}

\section{Article history:}

Received 15 January 2014

Accepted 16 August 2014

Available online 13 September 2014

\section{Keywords:}

MicroDMFC

Experimental studies

Modeling

Cell performance

\begin{abstract}
A B S T R A C T
The Direct Methanol Fuel Cell (DMFC) has attracted much attention due to its potential applications as a power source for transportation and portable electronic devices. Based on the advantages of the scaling laws, miniaturization promises higher efficiency and performance of power generating devices and the MicroDMFC is therefore an emergent technology. In this work, a set of experiments with a MicroDMFC of $2.25 \mathrm{~cm}^{2}$ active area are performed in order to investigate the effect of important operating parameters. Maximum power density achieved was $32 \mathrm{~mW} / \mathrm{cm}^{2}$ using a $4 \mathrm{M}$ methanol concentration at room temperature. Polarization curves are compared with mathematical model simulations in order to achieve a better understanding of how parameters affect performance. The one-dimensional model used in this work takes in account coupled heat and mass transfer, along with the electrochemical reactions occurring in a direct methanol fuel cell and was already developed and validated for DMFC in previous work by Oliveira et al. [1-3]. The model is also used to predict some important parameters to analyze fuel cell performance, such as water transport coefficient and methanol crossover. This easy to implement simplified model is suitable for use in real-time MicroDMFC simulations. More experimental data are also reported bearing in mind the insufficient experimental data available in literature at room temperature, a goal condition to use this technology in portable applications.
\end{abstract}

() 2014 Elsevier Ltd. All rights reserved.

\section{Introduction}

In the last few years, the growing interest in portable devices (laptop computers, 3G cellular phones and internet-enabled PDAs) has stimulated the research on miniaturized portable fuel cells to overcome batteries limitations [4]. Micro Fuel Cells can compete with batteries in the low power range $(0-30 \mathrm{~W})$. Mostly due to the lack of effective miniaturized hydrogen storage technologies, a liquid fuel like methanol could be the best option to reach a high power density with an attractive cost-to-power ratio. MicroDMFCs can also operate at ambient temperature, leading to a reduction on thermal management challenges for small systems. Small DMFCs with various degrees of microfabrication have been reported [5,6]. Regarding design parameters, the most common materials used as carrier substrate are silicon and stainless steel. Stainless steel leads to high fuel cell performances. Cha [7] used numerical simulation to compare the performance of several cell designs. The central part of

\footnotetext{
* Corresponding authors. Tel.: +351 225081675; fax: +351 225081449.

E-mail addresses: dfalcao@fe.up.pt (D.S. Falcão), apinto@fe.up.pt (A.M.F.R. Pinto).
}

the MicroDMFC is the proton exchange membrane. Methanol crossover across the membrane is one of the most important problems to solve $[8,9]$. High methanol concentration provides achievable energy density but it also causes severe methanol crossover through the membrane resulting in a mix potential at the cathode and could lead to lower cell performances. Concerning the different concepts of fuel delivery and handling, the MicroFCs are categorized as passive and active [10]. An active system needs moving parts to feed oxidant or fuel to the cell requiring power to operate. Channels configuration is crucial to an efficient reactants distribution for active MicroDMFCs. A passive cell requires no external power. The design optimization demands for a better understanding of the flow dynamics. The control of the multiphase flows at the microscale is a crucial issue. The $\mathrm{CO}_{2}$ bubbles formed at the anode can disturb and eventually block the flow. On the cathode side the water produced is injected into the channels and the developed two-phase flow plays a central role in fuel cell water management [11]. Both experimental and modeling studies are still needed to overcome or minimize the problems caused by an inadequate water and heat management. Despite the importance of water management in determining the MicroFC performance, no 